\title{
Topological Charge in SU(2) Lattice Gauge Theory
}

\author{
I. A. Fox, J. P. Gilchrist, M. L. Laursen, and G. Schierholz \\ Deutsches Elektronen-Synchrotron DESY, D-2000 Hamburg 52, Germany \\ (Received 10 September 1984)
}

\begin{abstract}
Using the expression for the topological charge proposed by Lüscher, we calculate the topological susceptibility in SU(2) lattice gauge theory. This problem has become tractable numerically because we were able to integrate one variable analytically. We verify the validity of this definition at present values of $\beta$ and compare our results to previous work.
\end{abstract}

PACS numbers: $11.15 . \mathrm{Ha}$

Differentiable non-Abelian SU $(N)$ gauge fields on a four-torus carry a topological charge ${ }^{1}$

$$
\begin{aligned}
& Q=-\frac{1}{16 \pi^{2}} \int d^{4} x \operatorname{tr}\left\{F_{\mu \nu} \tilde{F}_{\mu \nu}\right\}, \\
& \tilde{F}_{\mu \nu}=\frac{1}{2} \epsilon_{\mu \nu \rho \sigma} F_{\rho \sigma},
\end{aligned}
$$

which assumes integer values. It has been suggested that a nontrivial topological susceptibility

$$
\chi_{t}=\left\langle Q^{2}\right\rangle / v
$$

in QCD may solve its U(1) problem. ${ }^{2}$ Using large- $N$ expansions, Witten ${ }^{3}$ has shown that this quantity is related to the $\eta^{\prime}$ mass by ${ }^{4}$

$$
\chi_{t}=\frac{f_{\pi}^{2}}{2 N_{f}}\left(m_{\eta}^{2}+m_{\eta^{\prime}}^{2}-2 m_{K}^{2}\right) \underset{N_{f}=3}{=}(180 \mathrm{MeV})^{4},
$$

where $N_{f}$ is the number of massless flavors. The resolution of the $U(1)$ problem therefore reduces to showing that $\chi_{t}$ has the appropriate value. A nontrivial topological susceptibility may also be at the root of spontaneous chiral-symmetry breaking ${ }^{5}$ and possibly will lead to new phases (as a function of the vacuum angle $\theta$ ) such as "oblique confinement." It is therefore of great interest to compute $\chi_{t}$ by means of nonperturbative techniques. The lattice approach ${ }^{7}$ is one such technique and within this framework the problem has been studied by several authors. ${ }^{8-13}$

On the lattice, continuity in space is lost and it seems that one should be able to view any lattice field configuration as being a (discrete) copy of a smooth continuum configuration. This would always be topologically trivial since $F_{\mu \nu} \tilde{F}_{\mu \nu}=\partial_{\mu} J_{\mu}$ for some current $J_{\mu}$ and is therefore a total derivative. This is, however, not quite correct, at least in asymptotically free theories where the critical point is at zero coupling $(\beta \rightarrow \infty)$. In the continuum region, fluctuations in the Wilson loop $W$ at the scale of a lattice spacing $a$ are small. Furthermore, in this regime

$$
\langle W(a, a)\rangle=1-\frac{3}{2} \beta^{-1}+O\left(\beta^{-2}\right),
$$

so for large $\beta$, most of the Wilson loops will have values close to unity. We are justified, therefore, in interpolating the fields defined only on the links of the lattice to the whole volume by keeping the Wilson loops at and below the scale of $a$ near the identity. This may force us into introducing gauge singularities which will result in a nontrivial topological charge.

A lattice construction of $Q$, which appears to be the most natural (and geometrically straightforward), has been proposed by Lüscher ${ }^{9}$ for the gauge group SU(2). The central idea is that the information about the topology of the gauge field configuration is carried by a set of transition functions $v_{n, \mu}(x)$ defined on the cubes $f(n, \mu)$ forming the intersection of two adjacent hypercubes cornered at $n$ and $n-\hat{\mu}$. These transition functions are simply gauge transformations which relate the gauge fields within neighboring hypercubes. The explicit expression for $Q$ is 9,14

$$
\begin{aligned}
Q=-\frac{1}{24 \pi^{2}} \sum_{n} \sum_{\mu, \nu, \rho, \sigma} \epsilon_{\mu \nu \rho \sigma}\left\{3 \int_{p(n, \mu, \nu)} d^{2} x \operatorname{tr}\left[\left(v_{n, \mu} \partial_{\rho} v_{n, \mu}^{-1}\right)\left(v_{n-\hat{\mu}, \nu}^{-1} \partial_{\sigma} v_{n-\hat{\mu}, \nu}\right)\right]\right. \\
\left.+\int_{f(n, \mu)} d^{3} x \operatorname{tr}\left[\left(v_{n, \mu}^{-1} \partial_{\nu} v_{n, \mu}\right)\left(V_{n, \mu}^{-1} \partial_{\rho} v_{n, \mu}\right)\left(v_{n, \mu}^{-1} \partial_{\sigma} v_{n, \mu}\right)\right]\right\} .
\end{aligned}
$$

The sum is over all hypercubes and $p(n, \mu, \nu)$ is the plaquette forming the intersection of the four hypercubes cornered at $n, n-\hat{\mu}, n-\hat{\nu}$, and $n-\hat{\mu}-\hat{\nu}$. Equation (5) can be shown to have the following properties ${ }^{9}$ : (a) $Q$ is defined for all lattice fields except for a singular set of measure zero in the functional integral, so-called exceptional fields. (b) $Q$ takes integer values and does not change under continuous deformations of the field provided no exceptional config- urations are encountered. (c) $Q$ has a local form. (d) $Q$ reduces to the familiar expression Eq. (1) in the classical continuum limit. Property (b) in particular means that $Q$ is a genuine topological object $-\chi_{t}$ will contain no perturbative contributions.

As it stands, a numerical calculation of Eq. (5) would be very time consuming in practice (if not impossible on present day computers) since the transition 
functions are rather complicated and the integrals must be computed to a high precision. (We estimated $\sim 20$ hours for one smooth gauge field configuration on a $6^{4}$ lattice using a sequential computer.) In view of this, a more tractable but nonlocal definition of $Q$ on the lattice was recently suggested by Woit. ${ }^{13}$ One must pay a price, however-namely, it does not always properly reconstruct the topological charge for all field configurations. In the two-dimensional U(1) gauge theory, where Eq. (5) reduces to a sum over plaquettes, it has turned out ${ }^{15}$ that both definitions agree only occasionally, configuration by configuration. Furthermore, Woit's definition depends strongly on the choice of the time axis.

We circumvented the problem of time consumption in a different way: We performed, analytically, one integral of the volume term and one of the surface term thus bringing Eq. (5) into a form suitable for Monte Carlo evaluation. The calculation ${ }^{16}$ can be summarized briefly as follows: The transition functions involve products of powers $u^{y}$ where $0 \leqslant y \leqslant 1$ and the $u$ 's are parallel transporters along closed loops within the hypercubes cornered at $n$ and $n-\hat{\mu}$ and passing through the corner point. Typically we start from a product of the form

$$
\left(u_{p_{0} p_{2}}^{-1}\right)^{y}\left(u_{p_{0} p_{2}} u_{p_{2} p_{3}} u_{p_{1} p_{3}}^{-1} u_{p_{0} p_{1}}^{-1}\right)^{y} u_{p_{0} p_{1}}\left(u_{p_{1} p_{3}}\right)^{y}
$$

where $p_{0}, \ldots, p_{3}$ refer to the four corners of a plaquette. This function interpolates between $u_{p_{0} p_{1}}$ and $u_{p_{2} p_{3}}$. By essentially repeating this procedure, and introducing a new parameter each time, one obtains an explicit expression for the transition functions.

For computational reasons we have defined

$$
u^{y}=(1-y+y u) \operatorname{det}(1-y+y u)^{-1 / 2}
$$

for $0 \leqslant y \leqslant 1$ and $u \in S U(2), u \neq-1$. The differentiations within the integrand are next performed analytically and then, if we write $x=\left(x_{1}, x_{2}, x_{3}\right)$, the $x_{1}$ integrals reduce to

$$
\int_{0}^{1} d x_{1} \frac{x_{1}^{n}}{\operatorname{det}\left(1-x_{1}+x_{1} u\right)^{m}}
$$

for $n=0,1,2,3$, and $m=1,2,3$. These integrals are of standard type and can be performed analytically. Notice that only integer powers of the determinant will appear in the denominator so that after integration we end up with at most arctangents and rational functions. The expression for the once integrated function can be written in a fairly compact way, and is no worse than the original one. It is conceivable that even the integrals over $x_{2}$ can be done and we are investigating this presently.

We have performed a Monte Carlo calculation of the topological susceptibility Eq. (2) on a $5^{4}$ lattice at $\beta=2.2$ and on $6^{4}$ lattices at $\beta=2.3,2.4$, and 2.5 using the heat-bath algorithm. Before embarking on the calculation, we checked that the remaining integrals (which must be done numerically) can be carried out sufficiently accurately so as to allow an unambiguous assignment of an integer topological charge. To give an indication of the accuracy of our calculation, we state below the results of the first four consecutive "measurements" of $Q$ at $\beta=2.2$, demanding an accuracy of $0.03 \%$ in the numerical integrations:

$$
\begin{aligned}
& Q_{1}=1.00018 \pm 0.00262, \\
& Q_{2}=0.99999 \pm 0.00269, \\
& Q_{3}=-2.99969 \pm 0.00230, \\
& Q_{4}=-1.00012 \pm 0.00327 .
\end{aligned}
$$

On the average the area and volume terms are of the same magnitude, whereas in the naive continuum limit the area term dominates. Given that the contribution to $Q$ from each lattice point is typically of the order of 0.02 , we estimate that we could compute $Q$ unambiguously on lattices as large as $10^{4}$ under the same conditions. Our results for $a^{4} \chi_{t}=\left\langle Q^{2}\right\rangle / L^{4}$ are as follows:

$$
\begin{array}{cc}
\beta & \multicolumn{1}{c}{a^{4} \chi_{t}} \\
\hline 2.2 & (9.62 \pm 1.18) \times 10^{-3} \\
2.3 & (3.38 \pm 0.37) \times 10^{-3} \\
2.4 & (1.09 \pm 0.12) \times 10^{-3} \\
2.5 & (0.41 \pm 0.05) \times 10^{-3}
\end{array}
$$

Each entry in the table corresponds to "measurements" of $Q$ over more than 100 independent gaugefield configurations. Statistical errors are treated in the following manner. We divide the data sets into eight groups and for each group, we calculate the standard deviation $\sigma\left(\chi_{t}\right)$ on $X_{t}$. The error on the whole sample is then $\left(\Delta \chi_{t}\right)^{2}=\sigma^{2}\left(\chi_{t}\right) / 8$. The relatively large error at $\beta=2.5$ is connected with the fact that the charge distribution becomes increasingly narrow at larger $\beta$. If we assume the two-loop renormalization group formula for $a$,

$$
a(\beta)=\Lambda_{L}^{-1} \exp \left(-\frac{3 \pi^{2}}{11} \beta\right)\left(\frac{6 \pi^{2}}{11} \beta\right)^{51 / 121},
$$

this gives in units of $\Lambda_{L}$

$$
\begin{array}{cl}
\beta & 10^{-6} \Lambda_{L}^{-4} \chi_{t} \\
\hline 2.2 & 2.89 \pm 0.35 \\
2.3 & 2.76 \pm 0.30 \\
2.4 & 2.43 \pm 0.26 \\
2.5 & 2.51 \pm 0.29
\end{array}
$$

which is in fairly good agreement with asymptotic scaling.

We plot our data Eq. (10) together with the (fitted) 


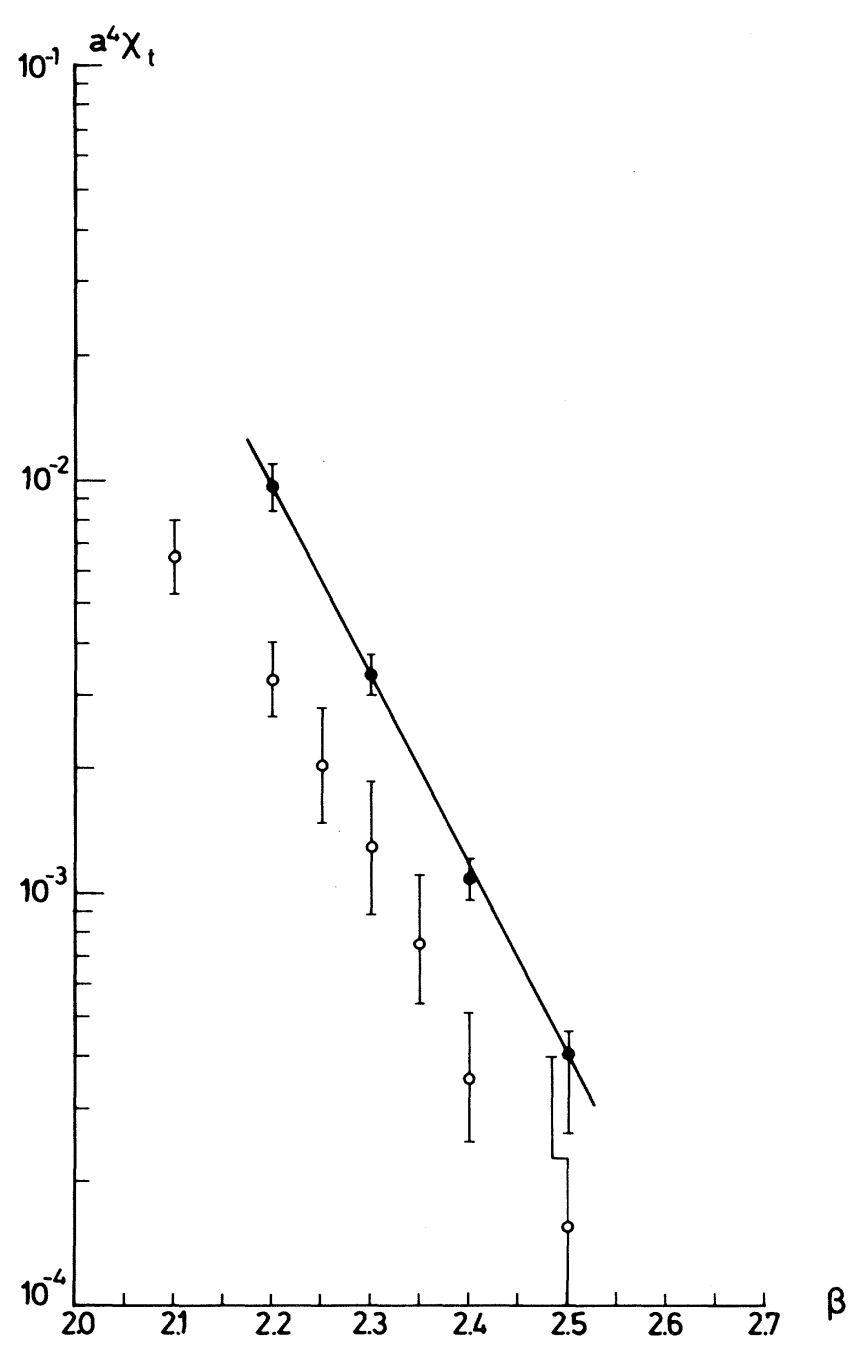

FIG. 1. The topological susceptibility $\chi_{t}$ in units of $a^{-4}$. The solid circles are the data points obtained in this work, the open circles are those of Woit. The line is the scaling curve fitted to our data.

scaling curve in Fig. 1. Taking now $\Lambda_{L} \cong 0.015 \sqrt{\sigma}$ at $\beta \sim 2.3$ (where we believe our lattice to be big enough) from averaging recent Monte Carlo calculations ${ }^{17}$ of the string tension $\sigma$ and assuming $\sqrt{\sigma}=400$ $\mathrm{MeV}$, we obtain for $\chi_{t}$

$$
\chi_{t}=(245 \pm 8 \mathrm{MeV})^{4}
$$

which is in the right ball-park. [It should be noted that Eq. (3) is not exact and that we are dealing with $N=2$ here.]

The observation of scaling (within errors) indicates that the lattice construction Eq. (5) of $Q$ is physically relevant at present values of $\beta$, i.e., it successfully mimics the topological properties of the continuum theory. On small lattices, such as ours, we would expect $\chi_{t}$ to fall below the scaling curve at larger values of $\beta$. This is presently masked by our large statistical error at $\beta=2.5$. To increase the statistics here, the strategy of Fucito and Solomon ${ }^{18}$ might prove useful.

In Fig. 1, we compare our results with those of Woit. ${ }^{13}$ We find that our values our $\chi_{t}$ lie consistently higher than his by a factor of about 2.5. This suggests that Woit's algorithm may have similar problems here as in the two-dimensional U(1) gauge theory. ${ }^{15}$

In the lattice version of the two-dimensional $\mathrm{O}(3) \sigma$ model, it was found that the topological susceptibility did not follow the expected scaling law. ${ }^{19}$ This unusual behavior was found to be due to short-range fluctuations of the topological charge 20 ("dislocations") with such a small action that they predominate over the contribution of slowly varying fields. Although in SU(2) gauge theory, no such large deviation of $\chi_{t}$ from the scaling curve is observed, it may be that "dislocations" still play some role. This remains to be investigated.

Another interesting calculation which we are currently looking at is the role played by topology in spontaneous chiral-symmetry breaking. ${ }^{21}$ This involves an investigation of the fermion zero modes and employs the Lanczos algorithm to find the eigenvalues of the fermion matrix and locate the fluctuations in the lowest ones.

We are grateful to $M$. Lüscher, C. Panagiotakopoulos, and M. Teper for useful discussions. Two of us (I.A.F. and J.P.G.) would like to thank the Science and Engineering Research Council and Royal Society for fellowships. We are indebted to the University of Hamburg for their generous allocation of computer time without which this work would not have been possible. Thanks are also due to $M$. Lüscher for a critical reading of the manuscript.

${ }^{1}$ A. A. Belavin, A. M. Polyakov, A. S. Schwartz, and Yu. S. Tyupkin, Phys. Lett. 59B, 85 (1975).

${ }^{2}$ G. 't Hooft, Phys. Rev. Lett. 37, 8 (1976), and Phys.

Rev. D 14, 3432 (1976).

${ }^{3}$ E. Witten, Nucl. Phys. B156, 269 (1979).

${ }^{4} \mathrm{G}$. Veneziano, Nucl. Phys. B159, 213 (1979).

${ }^{5} \mathrm{G}$. Veneziano, Phys. Lett. 95B, 90 (1980).

${ }^{6} \mathrm{G}$. 't Hooft, Nucl. Phys. B190, 455 (1981).

${ }^{7}$ K. G. Wilson, Phys. Rev. D 14, 2455 (1974).

8P. di Vecchia, K. Fabricius, G. C. Rossi, and G. Veneziano, Nucl. Phys. B192, 392 (1981).

${ }^{9}$ M. Lüscher, Commun. Math. Phys. 85, 29 (1982).

${ }^{10} \mathrm{~K}$. Ishikawa, G. Schierholz, H. Schneider, and M. Teper, Phys. Lett. 128B, 309 (1983).

11J. Polonyi, Phys. Rev. D 29, 716 (1984).

${ }^{12}$ Y. Iwasaki and T. Yoshiè, Phys. Lett. 131B, 159 (1983).

13P. Woit, Phys. Rev. Lett. 51, 638 (1982).

${ }^{14}$ P. van Baal, Commun. Math. Phys. 85, 529 (1982).

${ }^{15}$ C. Panagiotakopoulos, Rockefeller University Report No. $83 / \mathrm{B} / 85,1984$ (to be published). 
16I. A. Fox, J. P. Gilchrist, M. L. Laursen, and G. Schierholz, to be published.

${ }^{17} \mathrm{~F}$. Gutbrod and I. Montvay, Phys. Lett. 136B, 411 (1984); F. Karsch and C. B. Lang, Phys. Lett. 138B, 176 (1984).

${ }^{18} \mathrm{~F}$. Fucito and S. Solomon, California Institute of Technology Report No. CALT-68-1130, 1984 (to be published).
${ }^{19}$ B. Berg and M. Lüscher, Nucl. Phys. B190, 412 (1981).

${ }^{20}$ B. Berg, Phys. Lett. 104B, 475 (1981); M. Lüscher, Nucl. Phys. B200, 61 (1982); B. Berg and C. Panagiotakopoulos, Deutsches Elektronen Synchrotron Report No. 84-037, 1984 (to be published).

${ }^{21}$ I. M. Barbour, P. Gibbs, J. P. Gilchrist, H. Schneider, G. Schierholz, and M. Teper, Phys. Lett. 136B, 80 (1984). 